\title{
CHARACTERIZATIONS OF SCHÜTZENBERGER GRAPHS IN TERMS OF THEIR AUTOMORPHISM GROUPS AND FUNDAMENTAL GROUPS
}

\author{
by DAVID COWAN and NORMAN R. REILLY
}

(Received 17 May, 1991)

\begin{abstract}
The importance of the fundamental group of a graph in group theory has been well known for many years. The recent work of Meakin, Margolis and Stephen has shown how effective graph theoretic techniques can be in the study of word problems in inverse semigroups. Our goal here is to characterize those deterministic inverse word graphs that are Schützenberger graphs and consider how deterministic inverse word graphs and Schützenberger graphs can be constructed from subgroups of free groups.
\end{abstract}

1. Introduction and background. Let $X$ denote a finite non-empty set and $X^{-1}$ a set in one-to-one correspondence with $X$ (via $x \leftrightarrow x^{-1}$ ). We define a unary operation on the free semigroup $\left(X \cup X^{-1}\right)^{+}$inductively by the rules

$$
\begin{aligned}
& \left(x^{-1}\right)^{-1}=x \quad(x \in X) \\
& (a b)^{-1}=b^{-1} a^{-1} \quad\left(a \in\left(X \cup X^{-1}\right)^{+}, b \in X \cup X^{-1}\right) .
\end{aligned}
$$

In particular, we will have $\left(a^{-1}\right)^{-1}=a$, for all $a \in\left(X \cup X^{-1}\right)^{+}$.

By an inverse word graph over $X$ is meant a pair of sets $\Gamma=(V, E)$ where

(i) $E \subseteq V \times\left(X \cup X^{-1}\right) \times V$,

(ii) $(u, x, v) \in E \Rightarrow\left(v, x^{-1}, u\right) \in E$,

(iii) for any $u, v \in V$, there exists $\left(u_{i}, x_{i}, v_{i}\right) \in E, 0 \leq i \leq n$, with

$$
u=u_{0}, \quad v_{i}=u_{i+1} \quad(0 \leq i \leq n-1), \quad v_{n}=v .
$$

We describe condition (iii) by saying that the graph is connected. For any $(u, x, v) \in E$, we define the label of $(u, x, v)$ to be $x$. For general background information on graphs we refer the reader to Serre [8].

The inverse word graph $\Gamma=(V, E)$ is said to be deterministic if

(iv) $(u, x, v),(u, x, w) \in E$ implies that $v=w$

and to be injective if

(v) $(u, x, w),(v, x, w) \in E$ implies that $u=v$.

It is a simple exercise to show that an inverse word graph is deterministic if and only if it is injective. We adopt the usual conventions for representing graphs diagrammatically. In particular, we will only display edges labelled by elements of $X$ and it will be understood that to every displayed edge $(u, x, v)$ with a label from $X$ there is andisplayed edge $\left(v, x^{-1}, u\right)$ with a label from $X^{-1}$. The following is an example of a deterministic inverse word graph:

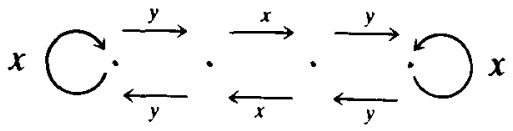

Deterministic inverse word graphs were introduced by J. B. Stephen as a tool in the study of word problems in inverse semigroups and have subsequently been employed with considerable success by Stephen [9], Margolis and Meakin [3], [4], [5], [6] and Margolis, 
Meakin and Jones [7]. Unless otherwise stated we adopt the notation and terminology of [1].

A presentation of an inverse semigroup $S$ is a pair $\langle X ; R\rangle$ where $X$ is a non-empty set and $R$ is a binary relation on $F \mathscr{I}(X)$, the free inverse semigroup on $X$, such that

$$
S \cong F \mathscr{F}(X) / \rho,
$$

where $\rho$ is the congruence on $F \mathscr{I}(X)$ generated by $R$. A presentation is said to be finite when both $X$ and $R$ are finite.

For any $w \in F \mathscr{\Phi}(X)$ and any congruence $\rho$ on $F \mathscr{\Phi}(X)$, define the Schützenberger graph $\Gamma_{\rho}(w)=(V, E)$ of $w$ relative to $\rho$ by:

$$
\begin{aligned}
& V=R_{w \rho}, \\
& E=\left\{(a \rho, x, b \rho): a \rho, b \rho \in R_{w \rho}, x \in X \cup X^{-1} \text { and } a x \rho=b \rho\right\} .
\end{aligned}
$$

Lemma 1.1. (Stephen [9], Theorem 3.1). $\Gamma_{p}(w)$ is a deterministic inverse word graph.

Let $\Gamma=(U, E), \Delta=(V, F)$ be two inverse word graphs over $X$. By an isomorphism of $\Gamma$ to $\Delta$ is meant a pair $(\varphi, \chi)$ of mappings such that

(i) $\varphi$ is a bijection of $U$ onto $V$, and

(ii) $\chi$ is a bijection of $E$ onto $F$ such that

$$
\chi(u, x, v)=(\varphi(u), x, \varphi(v)), \text { for all }(u, x, v) \in E .
$$

In other words, isomorphisms must preserve labels.

The importance of Schützenberger graphs in the study of inverse semigroup word problems derives from the next result.

TheOREM 1.2. (Stephen [9], Theorem 3.4). Let $u, v \in F \mathscr{F}(X)$ and $\rho$ be a congruence on $F \mathscr{I}(X)$.

(1) $u \rho \mathscr{L} v \rho \Leftrightarrow$ there exists an isomorphism of $\Gamma_{\rho}(u)$ onto $\Gamma_{\rho}(v)$ which maps u $\rho$ to $v \rho$. $v v^{-1} \rho$.

(2) $u \rho \mathscr{R} v \rho \Leftrightarrow$ there exists an isomorphism of $\Gamma_{\rho}(u)$ onto $\Gamma_{\rho}(v)$ which maps $u u^{-1} \rho$ to

(3) $u \rho \mathscr{D} v \rho \Leftrightarrow$ there exists an isomorphism of $\Gamma_{\rho}(u)$ onto $\Gamma_{\rho}(v)$.

(4) $u \rho \mathscr{H} v \rho \Leftrightarrow$ there exist isomorphisms of $\Gamma_{\rho}(u)$ onto $\Gamma_{\rho}(v)$ one of which maps u $\rho$ to $v \rho$, while the other maps $u u^{-1} \rho$ to $v v^{-1} \rho$.

(5) $u \rho=v \rho \Leftrightarrow$ there exists an isomorphism of $\Gamma_{\rho}(u)$ onto $\Gamma_{\rho}(v)$ which maps up to $v \rho$ and $u u^{-1} \rho$ to $v v^{-1} \rho$.

It is important to observe that Theorem 1.2(3) asserts that Schützenberger graphs are (to within isomorphism) associated with $\mathscr{D}$-classes. In particular, this enables us to identify any point in a Schützenberger graph with an idempotent as follows:

Lemma 1.3. (Stephen [9]). Let $u \in F \mathscr{I}(X)$ and $\rho$ be a congruence on $F \mathscr{F}(X)$. Let $P \in V(\Gamma(u))$ and $a$ be a word over $X \cup X^{-1}$ labelling a walk from $u u^{-1} \rho$ to $P$. Let $e=a^{-1} u u^{-1} a$. Then there exists an isomorphism of $\Gamma_{\rho}(u)$ to $\Gamma_{\rho}(e)$ mapping $P$ to $e$.

In order to turn Theorem 1.2 into an effective tool for solving word problems, Stephen developed a procedure for calculating the Schützenberger graphs of the form $\Gamma_{\rho}(u)$. For more details of this interesting technique, see Stephen [9].

2. Characterizing Schützenberger graphs. The question that we wish to consider in this section is which deterministic inverse word graphs are Schützenberger graphs. To do this we require some preliminaries. 
Lemma 2.1. Let $\Gamma$ be a deterministic inverse word graph over $X$ and $\rho$ denote the Vagner congruence on $\left(X \cup X^{-1}\right)^{+}$. Let $P \in V(\Gamma)$ and let $u, v \in\left(X \cup X^{-1}\right)^{+}$be such that $u \rho v$. If $u$ labels a walk from $P$ then so also does $v$.

Proof. Since $u \rho v$ it follows that $v$ can be obtained from $u$ by a sequence of elementary transitions of the form

$$
\left.\begin{array}{rl}
a p p^{-1} p b & \rightarrow a p b \\
a p p^{-1} q q^{-1} b & \rightarrow a q q^{-1} p p^{-1} b
\end{array}\right\}
$$

or their reverses. However it is clear that if a word on the left (respectively, right) in (1) labels a walk in $\Gamma$ from $P$ then so also does the corresponding word on the right (respectively, left). The result follows.

Although walks in a deterministic inverse word graph are labelled by words in $\left(X \cup X^{-1}\right)^{+}$, in the light of Lemma 2.1 we may consider them as being labelled by elements of $F \mathscr{S}(X)$.

We shall denote the symmetric inverse semigroup on a set $Y$ by $I(Y)$.

Lemma 2.2. Let $\Gamma$ be a deterministic inverse word graph over $X$. For each a $\in F \Phi(X)$ define a partial mapping $\theta_{a}=V(\Gamma) \rightarrow V(\Gamma)$ by:

$$
d\left(\theta_{a}\right)=\{v \in V(\Gamma) \text { : a labels a walk starting at } v\}
$$

and, for $v \in d\left(\theta_{a}\right)$,

$$
v \theta_{a}=w, \text { where a labels a walk from } v \text { to } w .
$$

Then $\theta: a \rightarrow \theta_{a}$ is a homomorphism of $F \Phi(X)$ into $I(V(\Gamma))$.

Proof. It follows from Lemma 2.1, that $d\left(\theta_{a}\right)$ is well defined for all $a \in F \mathscr{I}(X)$ and it follows from the assumption that $P$ is deterministic that the action of each $\theta_{a}$ is also well defined. It is straightforward to verify that $\theta$ is a homomorphism.

Definition 2.3. For any deterministic inverse word graph $\Gamma$ over $X$ let $\theta_{\Gamma}$ denote the homomorphism $\theta: F \mathscr{I}(X) \rightarrow I(V(\Gamma))$ defined in Lemma 2.2 and refer to $\theta_{\Gamma}$ as the natural representation of $F \mathscr{F}(X)$ in $I(V(\Gamma))$.

Lemma 2.4. Let $\Gamma$ be a deterministic inverse word graph and let $\theta: F \mathscr{I}(X) \rightarrow I(V(\Gamma))$ be the natural representation. For all automorphisms $\varphi$ of $\Gamma$ and all $a \in F \mathscr{H}(X)$,

$$
(\varphi(v)) \theta_{a}=\varphi\left(v \theta_{a}\right)
$$

Proof. This follows immediately from the definition of an isomorphism.

EXAmple 2.5 .

Clearly the graph $\Gamma$ depicted in Figure 1 is a deterministic inverse word graph. Here,

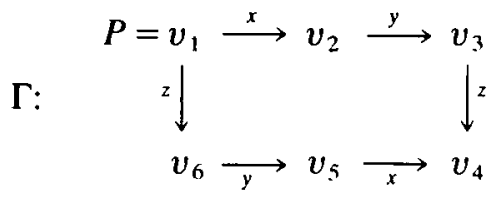

Figure 1. 
for example,

$$
d\left(\theta_{x}\right)=\left\{v_{1}, v_{5}\right\}, \quad v_{1} \theta_{x}=v_{2}, \quad v_{5} \theta_{x}=v_{4}
$$

with $\theta_{y}$ and $\theta_{z}$ being defined analogously.

Lemma 2.6. (Stephen, [9]). Let $\rho$ be a congruence on $F \mathscr{I}(X), e \in E(F \mathscr{I}(X))$ and $\Gamma=\Gamma_{\rho}(e)$. For each a $\in H_{e \rho}$, define a mapping $\lambda_{a \rho}: \Gamma \rightarrow \Gamma$ by

Then

$$
\lambda_{a \rho}(u \rho)=a u \rho \quad\left(u \rho \in R_{e \rho}\right) .
$$

$$
\lambda: a \rho \rightarrow \lambda_{a \rho} \quad\left(a \rho \in H_{e \rho}\right)
$$

is an isomorphism of $H_{e \rho}$ onto the automorphism group of $\Gamma$.

LemMA 2.7. Let $\Gamma$ be a Schützenberger graph over $X$. Let $w \in E(F \mathscr{F}(X))$ and $\rho$ be a congruence on $F \mathscr{I}(X)$ such that $\Gamma_{\rho}(w) \cong \Gamma$. Then $\rho \subseteq \theta_{\Gamma} \circ \theta_{\Gamma}^{-1}$.

Proof. Let $\theta=\theta_{\Gamma_{\rho}(w)}$. Clearly, $\theta_{\Gamma} \circ \theta_{\Gamma}^{-1}=\theta \circ \theta^{-1}$. Let $a, b \in F \mathscr{I}(X)$ be such that $(a, b) \in \rho$. Suppose that $a$ labels a walk from $u \rho$ to $v \rho$ for some $u \rho, v \rho \in R_{n \cdot \rho}$. Then $v \rho=u a \rho=u b \rho$ and so $b$ also labels a walk from $u \rho$ to $v \rho$. It follows that $\theta_{a} \subseteq \theta_{b}$. Similarly, $\theta_{b} \subseteq \theta_{a}$ and so $(a, b) \in \theta \circ \theta^{-1}=\theta_{\Gamma} \circ \theta_{\Gamma}^{-1}$.

Definition 2.8. Let $\Gamma$ be a deterministic inverse word graph over $X$ and let $w$ be a walk in $\Gamma$. Then we will denote by $\|w\|_{\lambda}$ the element of $F \mathscr{S}(X)$ corresponding to the word in $\left(X \cup X^{-1}\right)^{*}$ obtained by concatenating the labels of the edges in $w$.

TheOREM 2.9. Let $\Gamma$ be a finite deterministic inverse word graph, let $\Phi$ denote the automorphism group of $\Gamma$ and $P \in V(\Gamma)$. Let $\theta: F \mathscr{I}(X) \rightarrow I(V(\Gamma))$ be the natural representation. Then the following statements are equivalent.

(1) $\Gamma$ is a Schützenberger graph.

(2) There is an idempotent $e \in F \mathscr{I}(X)$ with $d\left(\theta_{e}\right)=\Phi(P)$, the orbit of $P$ under the action of $\boldsymbol{\Phi}$.

(3) For all $Q \in V(\Gamma) \backslash \Phi(P)$, there is a reduced word $w_{Q}$ such that $w_{Q}$ labels a walk from $P$ but does not label a walk from $Q$.

When (3) holds

$$
e=\prod\left\{w_{Q} w_{Q}^{-1}: Q \in V(\Gamma) \backslash \Phi(P)\right\}
$$

satisfies (2) and

$$
\Gamma \cong \Gamma_{\theta \circ \theta^{-1}}(e)
$$

Example 2.10. Let $\Gamma$ be as in Figure 1 and let $P=v_{1}$. It is easily seen that $\Phi$ is trivial. Then the elements

$$
w_{v_{2}}=w_{v_{3}}=w_{v_{4}}=w_{v_{6}}=x \text { and } w_{v_{5}}=z
$$

satisfy Theorem $2.9(3)$ so that $\Gamma \cong \Gamma_{\theta \circ H^{-1}}(e)$ where $e=x x^{-1} z z^{-1}$.

Proof of Theorem 2.9. (1) implies (2). Let $\rho$ be a congruence on $F \mathscr{I}(X)$. By Lemma 1.3 there exists $e \in E(F \mathscr{Y}(X))$ such that $\Gamma \cong \Gamma_{\rho}(e)$, with $e \rho$ corresponding to $P$. Then $V(\Gamma)=R_{e \rho}$. Let $S=F \mathscr{I}(X) / \rho$ and $a \in F \mathscr{I}(X)$. We have

$$
\begin{aligned}
a \rho \in H_{e \rho} & \Rightarrow(a \rho)(e \rho)=a \rho \\
& \Rightarrow a \rho \in d\left(\theta_{e}\right)
\end{aligned}
$$


so that $H_{e \rho} \subseteq d\left(\theta_{c}\right)$. On the other hand,

$$
\begin{aligned}
a \rho \in d\left(\theta_{e}\right) \Rightarrow & a \rho, a e \rho \in R_{e \rho} \\
\Rightarrow & a a^{-1} \rho=e \rho=a e a^{-1} \rho \\
\Rightarrow & a a^{-1} \rho=e \rho, \\
& a^{-1} a \rho=a^{-1} e a \rho=a^{-1} a e a^{-1} a \rho \leq e \rho .
\end{aligned}
$$

However, we cannot have $a^{-1} a \rho<e \rho=a a^{-1} \rho$ since then the $\mathscr{D}$-class of $e \rho$ would contain a copy of the bicyclic semigroup ([1], Lemma 1.31) contradicting the finiteness of $R_{e \rho}=V\left(\Gamma_{\rho}(e)\right)$. Hence $a^{-1} a \rho=e \rho=a a^{-1} \rho$ and $a \rho \in H_{e \rho}$. That (2) holds is now an easy consequence of Lemma 2.6.

(2) implies (1). Let $\rho=\theta \circ \theta^{-1}$. Define $\eta: R_{e \rho} \rightarrow V(\Gamma)$ by

$$
(u \rho) \eta=P \theta_{u} \quad\left(u \rho \in R_{c \rho}\right) .
$$

Since $\rho=\theta \circ \theta^{-1}, \eta$ is well defined.

Let $u \rho, v \rho \in R_{e \rho}$ and $(u \rho) \eta=(v \rho) \eta$. Then $P \theta_{u}=P \theta_{v}$. Since $u \rho, v \rho \in \mathscr{R}_{e \rho}$, it follows that $\theta_{u}, \theta_{v} \in R_{\theta_{r}}$ so that

$$
d\left(\theta_{u}\right)=d\left(\theta_{v}\right)=d\left(\theta_{c}\right)=\Phi(P) .
$$

Let $Q \in d\left(\theta_{u}\right)$. Then there exists an automorphism $\varphi$ of $\Gamma$ with $\varphi(P)=Q$. Consequently,

$$
\begin{aligned}
Q \theta_{v} & =(\varphi(P)) \theta_{v}=\varphi\left(P \theta_{v}\right) \quad \text { by Lemma } 2.4 \\
& =\varphi\left(P \theta_{u}\right)=(\varphi(P)) \theta_{u} \\
& =Q \theta_{u} .
\end{aligned}
$$

Thus $\theta_{u}=\theta_{v}$ and $\eta$ is one-to-one.

For any $Q \in V(\Gamma)$, there exists a walk $p$ from $P$ to $Q$. Let $u=\|p\|_{\lambda}$. Then $e \rho \in d \theta_{u}=d \theta_{u u^{-1}}$. By Lemma 2.4, this implies that

$$
d\left(\theta_{e}\right)=\Phi(P) \subseteq d\left(\theta_{u u^{-1}}\right) .
$$

Let $Q \in \Phi(P)$. Since $P \theta_{u u^{-1}}=P$, it again follows from Lemma 2.4 that $Q \theta_{u u^{-1}}=Q=Q \theta_{c}$. Therefore $\theta_{e} \leq \theta_{u u^{-1}}$ or $e \rho \leq u u^{-1} \rho$. Hence $e u \rho \in R_{e \rho}$. In addition, $e u$ also labels a walk from $P$ to $Q$ so that $P \theta_{e u}=Q$ and $(e u \rho) \eta=Q$. Thus $\eta$ is a bijection of $V\left(\Gamma_{\rho}(e)\right)=R_{e \rho}$ onto $V(\Gamma)$. We extend $\eta$ to a mapping of $E\left(\Gamma_{\rho}(e)\right)$ into $E(\Gamma)$ by

$$
(u \rho, x, u x \rho) \eta=\left(P \theta_{u}, x, P \theta_{u x}\right) \quad\left(u \rho, u x \rho \in R_{e \rho}\right) .
$$

Note that

$$
\begin{aligned}
u \rho, u x \rho \in R_{e \rho} & \Rightarrow \theta_{u}, \theta_{u x} \in R_{\theta_{e}} \\
& \Rightarrow d\left(\theta_{u}\right)=d\left(\theta_{u x}\right)=d\left(\theta_{e}\right) \\
& \Rightarrow\left(P \theta_{u}, x, P \theta_{u x}\right) \in E(\Gamma),
\end{aligned}
$$

whence the extension of $\eta$ to $E\left(\Gamma_{\rho}(e)\right)$ is well defined.

Let $(Q, x, R) \in E(\Gamma)$. Let $u \rho \in R_{e \rho}$ be such that $Q=(u \rho) \eta=P \theta_{u}$. Then

$$
R=Q \theta_{x}=P \theta_{u} \theta_{x}=P \theta_{u x} .
$$

The next step is to show that $u x \rho \in R_{e \rho}$. Clearly $d\left(\theta_{u x}\right) \subseteq d\left(\theta_{u}\right)$. In order to establish 
equality, let $P^{\prime} \in d\left(\theta_{u}\right)=d\left(\theta_{e}\right)$. By hypothesis, there exists an automorphism $\varphi$ of $\Gamma$ with $\varphi(P)=P^{\prime}$. Let $\varphi(Q)=Q^{\prime}$. Then there exists $R^{\prime} \in V(\Gamma)$ with

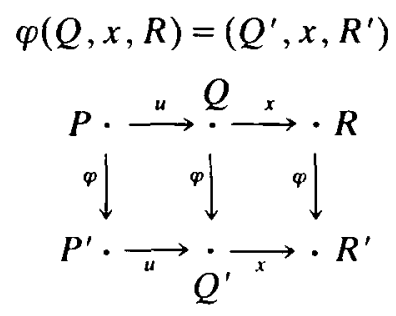

Hence

$$
P^{\prime} \theta_{u}=(\varphi(P)) \theta_{u}=\varphi\left((P) \theta_{u}\right)=\varphi(Q)=Q^{\prime},
$$

where $Q^{\prime} \in d\left(\theta_{x}\right)$. Thus $P^{\prime} \in d\left(\theta_{u} \theta_{x}\right)=d\left(\theta_{u x}\right)$, as required, and $d\left(\theta_{u x}\right)=d\left(\theta_{u}\right)=d\left(\theta_{e}\right)$. Consequently, $\theta_{u x} \in R_{\theta_{e}}$ so that $u x \rho \in R_{e \rho}$. Therefore

Moreover,

$$
(u \rho, x, u x \rho) \in E\left(\Gamma_{\rho}(e)\right) \text {. }
$$

$$
(u \rho, x, u x \rho) \eta=\left(P \theta_{u}, x, P \theta_{u x}\right)=(Q, x, R),
$$

whence $\eta$ maps $E\left(\Gamma_{\rho}(e)\right)$ onto $E(\Gamma)$. Finally, for $u \rho, u x \rho, v \rho, v y \rho \in R_{e \rho}$,

$$
\begin{aligned}
(u \rho, x, u x \rho) \eta=(v \rho, y, v y \rho) \eta & \Rightarrow(u \rho) \eta=(v \rho) \eta, x=y \\
& \Rightarrow u \rho=v \rho, x=y, \text { since } \eta \text { is injective on } R_{e p} \\
& \Rightarrow(u \rho, x, u x \rho)=(v \rho, y, v y \rho),
\end{aligned}
$$

whence $\eta$ is a bijection and an isomorphism. This completes the proof that (2) implies (1).

(2) implies (3). Let $e=u_{1} u_{1}^{-1} \ldots u_{n} u_{n}^{-1}$ where each $u_{i}$ is a reduced word. Then

$$
d\left(\theta_{e}\right)=\bigcap\left\{d\left(\theta_{u_{i}}\right): i=1, \ldots, n\right\}
$$

and (3) follows from (2).

Now let (3) hold and let

$$
e=\prod\left\{w_{Q} w_{Q}^{-1}: Q \in V(\Gamma) \backslash \Phi(P)\right\} .
$$

Then $P \in d\left(\theta_{e}\right)$ so that, by Lemma $2.4, \Phi(P) \subseteq d\left(\theta_{e}\right)$. On the other hand, it is clear that $Q \notin \Phi(P)$ implies that $Q \notin d\left(\theta_{e}\right)$. Thus

and (2) holds.

$$
d\left(\theta_{e}\right)=\Phi(P)
$$

EXAMPLE 2.11. Let $\Gamma$ be the graph depicted in Figure 2.

Clearly $\Gamma$ is a deterministic inverse word graph over $X=\{x, y\}$ with trivial automorphism group $\Phi$. Hence $\Phi(P)=P$. However, since there are edges labelled $x, x^{-1}, y$ and $y^{-1}$

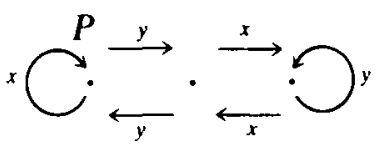

Figure 2. 
from every vertex, all three vertices are in the domain of every element of $F \mathscr{I}(X) \theta$. Thus Theorem 2.9(2) is not satisfied and $\Gamma$ is not a Schützenberger graph.

Example 2.12. Every finite deterministic inverse word graph over a singleton set $X=\{x\}$ is either a linear graph or a cycle. In either case, Theorem 2.9(2) is easily verified so that all such graphs are Schützenberger graphs.

EXAMPLE 2.13. Let $\Gamma$ be a finite deterministic inverse word graph over $X$ such that, for all $x \in X$, the subgraph of $\Gamma$ consisting of those edges labelled by $x$ is a connected linear graph. Then $\Gamma$ is a Schützenberger graph.

3. Finite presentations. Much of the interest in Schützenberger graphs derives from the fact that they can be used in studying word problems for finitely presented inverse semigroups. It is therefore natural to consider whether or not every finite Schützenberger graph actually arises in association with a finite presentation.

Theorem 3.1. Let $\Gamma$ be a finite Schützenberger graph over $X$. Then there exist a word $w$ and a finite presentation $S=\langle X ; R\rangle$ with corresponding congruence $\tau$ such that

(i) $\Gamma \cong \Gamma_{\tau}(w)$;

(ii) $\tau$ is the least congruence $\rho$ such that $\Gamma \cong \Gamma_{\rho}(w)$.

Proof. First of all, let

$$
w=\prod\left\{w_{Q} w_{Q}^{-1}: Q \in V(\Gamma) \backslash \Phi(P)\right\},
$$

where $P$ is a distinguished vertex of $\Gamma$ and, for each $Q \in V(\Gamma) \backslash \Phi(P), w_{Q}$ is a reduced word that labels a walk in $\Gamma$ starting at $P$ but does not label a walk in $\Gamma$ starting at $Q$. By Theorem 2.9 and its proof, there exists a congruence $\rho$, say, on $F \mathscr{G}(X)$ with $\Gamma \cong \Gamma_{\rho}(w)$ and with $P$ corresponding to $w \rho$ under the isomorphism.

Let $T$ be a spanning tree of $\Gamma$ and, for each $v \in V(\Gamma)$, denote by $g_{v}$ the word that labels the geodesic in $T$ from $P$ to $v$.

Let $R=R_{1} \cup R_{2}$, where

and

$$
R_{1}=\left\{\left(w, w g_{v} g_{v}^{-1}\right): v \in V(\Gamma)\right\}
$$

$$
R_{2}=\left\{\left(w, w g_{v_{1}} x g_{v_{2}}^{-1}\right):\left(v_{1}, x, v_{2}\right) \in E(\Gamma \backslash T)\right\} .
$$

Let $S=\langle X ; R\rangle$ and $\tau$ be the congruence on $F \mathscr{I}(X)$ generated by $R$. Since $\Gamma$ is a finite graph, $\langle X ; R\rangle$ is a finite presentation for $S$. Note that $w \tau=w^{2} \tau$.

Observe that if $\Gamma \cong \Gamma_{\sigma}(w)$ for some congruence $\sigma$ then $R \subseteq \sigma$, by the definition of Schützenberger graph, so that $\tau \subseteq \sigma$. Thus, if we can establish statement (i) of the theorem, then statement (ii) follows immediately.

Define a mapping $\phi: \Gamma \rightarrow \Gamma_{\tau}(w)$ by

and

$$
v \phi=w g_{v} \tau \quad(v \in V(\Gamma))
$$

$$
\left(v_{1}, x, v_{2}\right) \phi=\left(v_{1} \phi, x, v_{2} \phi\right) \quad\left(\left(v_{1}, x, v_{2}\right) \in E(\Gamma)\right) .
$$

We first show that $\phi$ maps $\Gamma$ into $\Gamma_{\tau}(w)$. For any $v \in V(\Gamma),\left(w, w g_{v} g_{v}^{-1}\right) \in R$ and so $w \tau \mathscr{R} w g_{v}, \tau$. Thus, $\phi$ maps $V(\Gamma)$ into $V\left(\Gamma_{\tau}(w)\right)$. Next, let $\left(v_{1}, x, v_{2}\right) \in E(\Gamma)$. We consider two cases:

Case 1: $\left(v_{1}, x, v_{2}\right)$ is an edge of $T$. Then either $g_{v_{1}} x=g_{v_{2}}$ or $g_{v_{2}} x^{-1}=g_{v_{1}}$. If $g_{v_{1}} x=g_{v_{2}}$ 
then $w g_{v_{1}} \tau x \tau=w g_{v_{2}} \tau$ and $\left(w g_{v_{1}} \tau, x, w g_{v_{2}} \tau\right)=\left(v_{1} \phi, x, v_{2} \phi\right) \in E\left(\Gamma_{\tau}(w)\right)$. If $g_{v_{2}} x^{-1}=g_{v_{1}}$ then $w g_{v_{2}} x^{-1} \tau=w g_{v_{1}} \tau$, and so $\left(w g_{v_{2}} \tau, x^{-1}, w g_{v_{1}} \tau\right) \in E\left(\Gamma_{\tau}(w)\right)$, whence $\left(w g_{v_{1}} \tau, x, w g_{v_{2}} \tau\right)=$ $\left(v_{1} \phi, x, v_{2} \phi\right) \in E\left(\Gamma_{\tau}(w)\right)$.

Case 2: $\left(v_{1}, x, v_{2}\right)$ is not an edge of $T$. In this case, $w \tau w g_{v_{1}} x g_{v_{2}}^{-1}$ by the relations of $R_{2}$. Therefore,

$$
w g_{v_{2}} \tau w g_{v_{1}} x g_{v_{2}}^{-1} g_{v_{2}} \text {. }
$$

In any inverse semigroup, if $w b a^{-1} a=w a \mathscr{R} w$ then

and

$$
w=w w^{-1} w=\left(w b a^{-1} a\right)\left(w b a^{-1} a\right)^{-1} w=w b a^{-1} a b^{-1}
$$

$$
w a=w b a^{-1} a=w b a^{-1} a b^{-1} b=w b .
$$

Thus, by (2) with $b=g_{v_{1}} x$ and $a=g_{v_{2}}$ we have that

$$
w g_{v_{1}} x \tau w g_{v_{2}} .
$$

Therefore $\left(w g_{v_{1}} \tau, x, w g_{v_{2}} \tau\right) \in E\left(\Gamma_{\tau}(w)\right)$.

Consequently, $\phi$ is a well-defined graph map of $\Gamma$ into $\Gamma_{\tau}(w)$ which preserves incidence and orientation. In other words, $\phi$ is a graph morphism.

Suppose that $v_{1} \phi=v_{2} \phi$. Then $w g_{v_{1}} \tau w g_{v_{2}}$. Since $\Gamma \cong \Gamma_{p}(w)$ and $\tau \subseteq \rho$, we must have $w g_{v_{1}} \rho w g_{v_{2}}$. It then follows that $g_{v_{1}}$ and $g_{v_{2}}$ label walks in $\Gamma_{\rho}(w)$ from $w \rho$ to the same vertex; that is $g_{v_{1}}$ and $g_{v_{2}}$ label coterminal walks in $\Gamma_{\rho}(w)$ from $w \rho$. Therefore, $g_{v_{1}}$ and $g_{v_{2}}$ label coterminal walks in $\Gamma$ from $P$. Consequently, $v_{1}=v_{2}$ and $\phi$ is one-to-one on the vertices of $\Gamma$ and hence, one-to-one on the edges of $\Gamma$. It remains to show that $\phi$ is surjective.

Towards a contradiction, suppose that $\phi$ is not surjective on the vertices of $\Gamma$. Among those vertices $Q$ not in the image of $\phi$, there is at least one that is joined by an edge to a vertex in the image of $\phi$. Assume that $Q$ is such a vertex. That is, suppose that

Then

$$
\left(w g_{v} \tau, x, Q\right) \in E\left(\Gamma_{\tau}(w)\right) \text {. }
$$

and since $\tau \subseteq \rho$,

$$
Q=w g_{v} x \tau \mathscr{R} w \tau,
$$

$$
w g_{v} x \rho \mathscr{R} w \rho .
$$

Thus there is an edge $\left(v, x, v^{\prime}\right) \in E(\Gamma)$ and we claim that $v^{\prime} \phi=Q$.

If $\left(v, x, v^{\prime}\right) \in E(T)$, then $g_{v} x=g_{v^{\prime}}$ or $g_{v^{\prime}} x^{-1}=g_{v}$. But then, either

$$
Q=w g_{v} x \tau=w g_{v}, \tau=v^{\prime} \phi
$$

in the first case, or

$$
Q=w g_{v} x \tau=w g_{v^{\prime}} x^{-1} x \tau=w g_{v^{\prime}} x^{-1} x g_{v^{\prime}}^{-1} g_{v^{\prime}} \tau=w g_{v} g_{v}^{-1} g_{v^{\prime}} \tau=w g_{v^{\prime}} \tau=v^{\prime} \phi
$$

in the latter case. In any event, $Q$ is the image of $\phi$, a contradiction.

If $\left(v, x, v^{\prime}\right) \notin E(T)$ then $w g_{v} x g_{v^{\prime}}^{-1} \tau w$, by the relations of $R_{2}$. As above, this implies that $w g_{v} x \tau w g_{v^{\prime}}$. But then $Q=w g_{v} x \tau=w g_{v^{\prime}} \tau=v^{\prime} \phi$, again a contradiction. Thus $\phi$ is surjective on the vertices of $\Gamma$.

Finally, let $\left(w g_{v_{1}} \tau, x, w g_{v_{2}} \tau\right)$ be an edge of $\Gamma_{\tau}(w)$. Then $w g_{v_{1}} x \tau w g_{v_{2}}$ and, since $\tau \subseteq \rho$, it follows that $w g_{v_{1}} x \rho w g_{v_{2}}$. Therefore $\left(v_{1}, x, v_{2}\right)$ is an edge of $\Gamma$ and $\phi$ maps this edge to $\left(w g_{v_{1}} \tau, x, w g_{v_{2}} \tau\right)$ in $E\left(\Gamma_{\tau}(w)\right)$. Thus, $\phi$ is surjective on the edges of $\Gamma$, whence $\phi$ is an isomorphism of $\Gamma$ onto $\Gamma_{r}(w)$. 
NotE. The congruence $\tau$ produced in Theorem 3.1 is not minimum in an absolute sense. In other words, there could be another word $v$ and presentation $P^{\prime}=\langle X ; S\rangle$ with the corresponding congruence $\sigma$ such that $\Gamma \cong \Gamma_{\sigma}(v)$ and where $\sigma$ is properly contained in $\tau$.

4. Fundamental groups. Let $\Gamma=(V, E)$ be a deterministic inverse word graph over $X$ and let $P \in V$. Let $\pi(\Gamma, P)=\left\{\right.$ reduced or empty words $w$ over $X \cup X^{-1}: w$ labels a walk in $\Gamma$ from $P$ to $P$ \}. Then it is easily seen that $\pi(\Gamma, P)$ is a subgroup of $F \mathscr{G}(X)$, the free group on $X$ and $\pi(\Gamma, P)$ is known as the fundamental group of $\Gamma$ at $P$. For the graph in Figure 1 , for instance, we would have

$$
x y z x^{-1} y^{-1} z^{-1} \in \pi(\Gamma, P) .
$$

For more background information on the fundamental groups of graphs, the reader is referred to Cohen [2] and Serre [8].

Let $\Phi$ denote the automorphism group of $\Gamma$ and define equivalence relations $\tau_{V}, \tau_{E}$ on $V$ and $E$ by

$$
\begin{gathered}
u \tau_{V} v \Leftrightarrow v=\varphi(u), \quad \text { for some } \quad \varphi \in \Phi \\
(u, x, v) \tau_{E}(p, y, q) \Leftrightarrow x=y \text { and there exists } \varphi \in \Phi \text { such that } \\
\varphi(u)=p, \quad \varphi(v)=q .
\end{gathered}
$$

Thus the equivalence classes of $\tau_{V}$ and $\tau_{E}$ are simply the orbits of $V$ and $E$ under the action of $\Phi$. It is straightforward to verify that

$$
\Gamma / \Phi:=\left(V / \tau_{V}, E / \tau_{E}\right)
$$

is a deterministic inverse word graph. We will refer to $\tau_{V}$ and $\tau_{E}$ as the orbit relations on $V$ and $E$, respectively, under $\Phi$.

It should be noted that the fundamental group that we describe here is slightly different from the standard fundamental group of an unlabelled graph. However, we can use the standard formulation to provide a set of free generators for $\pi(\Gamma, P)$, for a deterministic inverse word graph $\Gamma=(V, E)$ over $X$.

Let $E^{+}$denote the set of edges in $E$ of the form $(u, x, v)$, with $x \in X$, and, for any $(u, x, v) \in E$, write $(u, x, v)^{-1}=\left(v, x^{-1}, u\right)$. Then the standard fundamental group $\pi_{1}(\Gamma, P)$ consists of all elements in $F \mathscr{G}\left(E^{+}\right)$of the form

where

$$
e_{1} \ldots e_{n} \quad\left(e_{i} \in E^{+} \cup\left(E^{+}\right)^{-1}\right)
$$

$$
e_{i}=\left(u_{i}, x_{i}, u_{i+1}\right), \quad u_{1}=u_{n+1}=P .
$$

The next result is a special case of (Serre [8], Theorem 20).

Theorem 4.1. Let $\Gamma=(V, E)$ be a finite inverse word graph and $P \in V$. Let $T$ be a spanning tree for $\Gamma$. For each $y=(u, x, v) \in E(\Gamma \backslash T)$, let

$$
y^{\prime}=y_{1} \ldots y_{i} y y_{i+1} \ldots y_{n},
$$

where $y_{1}, \ldots, y_{i}$ are the edges of the geodesic from $P$ to $u$ and $y_{i+1}, \ldots, y_{n}$ are the edges of the geodesic from $v$ back to $P$. Then $\left\{y^{\prime}: y \in E(\Gamma \backslash T)\right\}$ is a set of free generators for $\pi_{1}(\Gamma, P)$. 
Now let $\lambda$ denote the "labelling" function defined by

$$
\lambda(u, x, v)=x \quad((u, x, v) \in E)
$$

where $x \in X \cup X^{-1}$. Then $\lambda$ extends to a unique homomorphism of $F \mathscr{G}\left(E^{+}\right)$onto $F \mathscr{G}(X)$, which we will also denote by $\lambda$. Clearly $\lambda$ maps $\pi_{1}(\Gamma, P)$ onto $\pi(\Gamma, P)$.

Lemma 4.2. $(\operatorname{ker} \lambda) \cap \pi_{1}(\Gamma, P)=\{e\}$.

Proof. Let $w \in \pi_{1}(\Gamma, P), w \neq e$ and let

$$
w=w_{1} \ldots w_{m} \quad\left(w_{i} \in E\right)
$$

in reduced form. Note that to say that $w$ is given in reduced form in (3) is equivalent to saying that the walk determined by $w$ has no backtracking. Then

$$
\lambda(w)=\lambda\left(w_{1}\right) \ldots \lambda\left(w_{m}\right) .
$$

Suppose that $\lambda\left(w_{i}\right)=\lambda\left(w_{i+1}\right)^{-1}$. Then we must have

$$
w_{i}=\left(u_{i}, x, u_{i+1}\right), \quad w_{i+1}=\left(u_{i+1}, x^{-1}, u_{i+2}\right),
$$

for some $u_{i}, u_{i+1}, u_{i+2} \in V, x \in X \cup X^{-1}$. But if $\left(u_{i}, x, u_{i+1}\right) \in E$ then $\left(u_{i+1}, x^{-1}, u_{i}\right) \in E$ and, since $\Gamma$ is deterministic, we must have $u_{i+2}=u_{i}$. But then $w_{i+1}=w_{i}^{-1}$ which contradicts the assumption that the word in (3) is reduced as written. Therefore $\lambda(w)$ is reduced as written in (4) so that if $w \neq e$ then necessarily $\lambda(w) \neq e$. The claim then follows.

Corollary 4.3. Let $\Gamma=(V, E)$ be a deterministic inverse word graph over $X$. Let $P \in V$ and $T$ be a spanning tree. For each $v \in V$, let $g_{v}$ be the word labelling the geodesic from $P$ to $v$. Then $\pi(\Gamma, P)$ is freely generated by

$$
\left\{g_{u} x g_{v}^{-1}:(u, x, v) \in E(\Gamma \backslash T), x \in X\right) .
$$

Proof. Since ker $\lambda$ has trivial intersection with $\pi_{1}(\Gamma, P)$, it follows that $\lambda$ is one-to-one on $\pi_{1}(\Gamma, P)$ and therefore an isomorphism of $\pi_{1}(\Gamma, P)$ onto $\pi(\Gamma, P)$. Hence $\lambda$ maps any set of free generators of $\pi_{1}(\Gamma, P)$ onto a set of free generators of $\pi(\Gamma, P)$. Since the set in (5) is the image under $\lambda$ of the set described in Theorem 4.1, the claim holds.

EXAMPLE 4.4. Let $\Gamma$ be as in Figure 2. As a spanning tree we may take

$$
P \cdot \stackrel{y}{\rightarrow} \cdot \stackrel{x}{\rightarrow} \cdot
$$

(together with the inverses of these edges). Then we obtain the following set of free generators for $\pi(\Gamma, P)$ :

$$
\left\{x, y^{2}, y x^{2} y^{-1}, y x y x^{-1} y^{-1}\right\} .
$$

Another important group in relation to a graph is its automorphism group. In the remainder of this section we present some curious interactions between the automorphism group and the fundamental group of a deterministic inverse word graph.

Lemma 4.5. Let $\Gamma=(V, E)$ be a deterministic inverse word graph and let $\Phi$ denote the automorphism group of $\Gamma$. Then the action of $\Phi$ on $\Gamma$ is semiregular (that is, if $\varphi \in \Phi$ and $P \in V$ are such that $\varphi(P)=P$, then $\varphi$ is the identity automorphism). 
Proof. Let $\varphi$ and $P$ be as in the statement and $Q \in V$. Since $\Gamma$ is connected there exists a sequence of edges $\left\{\left(v_{i}, x_{i}, v_{i+1}\right): i=0, \ldots, n\right\}$ with $P=v_{0}, Q=v_{n+1}$. Then

$$
\begin{aligned}
\varphi\left(v_{0}, x_{0}, v_{1}\right) & =\left(\varphi(P), x_{0}, \varphi\left(v_{1}\right)\right) \\
& =\left(P, x_{0}, \varphi\left(v_{1}\right)\right) \\
& =\left(v_{0}, x_{0}, \varphi\left(v_{1}\right)\right) .
\end{aligned}
$$

Since $\Gamma$ is deterministic, we must have $\varphi\left(v_{1}\right)=v_{1}$. A simple induction argument will now show that $\varphi\left(v_{i}\right)=v_{i}, i=0, \ldots, n+1$, so that $\varphi(Q)=Q$ and the claim holds.

THEOREM 4.6. Let $\Gamma=(V, E)$ be a deterministic inverse word graph over $X$ with automorphism group $\Phi$. Let $\tau_{V}$ be the orbit relation on $V$ under $\Phi$ and $P \in V$. Then

(i) $\pi(\Gamma, P)$ is a normal subgroup of $\pi\left(\Gamma / \Phi, P \tau_{V}\right)$,

(ii) $\Phi \cong \pi\left(\Gamma / \Phi, P \tau_{V}\right) / \pi(\Gamma, P)$.

Proof. (i) That $\pi(\Gamma, P)$ is a subgroup of $\pi\left(\Gamma / \Phi, P \tau_{V}\right)$ is clear. The normality of $\pi(\Gamma, P)$ will follow from the proof of (ii).

(ii) Let $H=\pi\left(\Gamma / \Phi, P \tau_{V}\right)$ and $g \in H$. Then $g$, in reduced form, labels a walk in $\Gamma / \Phi$ from $P \tau_{V}$ to $P \tau_{V}$ so that, in $\Gamma, g$ must label a walk from $P$ to some element $Q$ in $P \tau_{V}$. But $P \tau_{V}=\Phi(P)$ and so there must exist an automorphism $\varphi_{g}$ of $\Gamma$ mapping $P$ to $Q$. By Lemma $4.5, \varphi_{g}$ is unique and so the mapping

$$
\varphi: g \rightarrow \varphi_{g} \quad(g \in H)
$$

is well defined. Now let $h$ be another element of $H$ and let $h$ label a walk from $P$ to $R$. The automorphism $\varphi_{g}$ must translate this to a walk labelled by $h$ from $Q$ to $S$, say, where $S=\varphi_{g}(R)$.

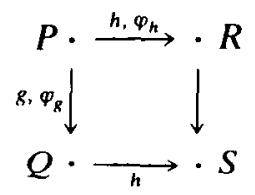

Then $g h$ labels a walk from $P$ to $S$ so that $\varphi_{g^{\prime}}$ is the unique automorphism of $\Gamma$ mapping $P$ to $S$. But

$$
\varphi_{g} \varphi_{h}(P)=\varphi_{g}(R)=S,
$$

whence $\varphi_{g} \varphi_{h}=\varphi_{g h}$ and $\varphi$ is a homomorphism.

Now consider any $\theta \in \Phi$ and let $\theta(P)=Q$. Since $\Gamma$ is connected, there exists a reduced word $g$ labelling a walk from $P$ to $Q$. Then $g \in H$ and $\varphi_{g}$ is an automorphism mapping $P$ to $Q$. By Lemma 4.5, $\varphi_{g}=\theta$ and so $\varphi$ is an epimorphism.

Finally, it is clear that the kernel of $\varphi$ is exactly $\pi(\Gamma, P)$. Therefore (ii) holds, by the first isomorphism theorem for groups.

5. Construction of deterministic inverse word graphs. In this section we describe a general technique for constructing deterministic inverse word graphs and Schützenberger graphs from subgroups of free groups and we will show how this construction relates to the fundamental groups of such graphs. 
Definition 5.1. Let $L$ be a subset of $F \mathscr{G}(X)$ containing 1 and with the following property. If $x_{1} \ldots x_{n+1} \in L$, where $x_{i} \in X \cup X^{-1}(i=1, \ldots, n+1)$ and $x_{1} \ldots x_{n+1}$ is a reduced word, then $x_{1} \ldots x_{n} \in L$. We shall call such a set a Schreier subset or say that it has the Schreier property.

When discussing Schreier subsets, it is convenient to view $F \mathscr{G}(X)$ as consisting of reduced words.

Construction. Let $N$ be a subgroup of $F \mathscr{G}(X)$ and $L$ a Schreier subset of $F \mathscr{G}(X)$ which is also the union of a finite number of cosets of $N$. Put

$$
\begin{aligned}
& V_{N, L}=\{N a: a \in L\}, \\
& E_{N, L}=\left\{(N a, x, N a x): N a, N a x \in V_{N, L}, x \in X \cup X^{-1}\right\}, \\
& \Gamma_{N, L}=\left(V_{N, L}, E_{N, L}\right) .
\end{aligned}
$$

When $N$ and $L$ are related as above, we shall refer to $(N, L)$ as an inverse word pair.

THEOREM 5.2. $\Gamma_{N . L}$ is a finite deterministic inverse word graph over $X$ and every finite deterministic inverse word graph over $X$ is isomorphic to one of this form.

Proof. Condition (i) for $\Gamma_{N . L}$ to be an inverse word graph is satisfied by the definition of $E_{N, L}$. In regard to condition (ii), let $(N a, x, N a x) \in E_{N, L}$, where $x \in X \cup X^{-1}$ and we may assume that $a$ is reduced. Then $a$ and the reduced form of $a x$ both belong to $L$. Hence the reduced forms of $a x$ and $(a x) x^{-1}=a$ belong to $L$ so that $\left(N a x, x^{-1}, N a\right) \in$ $E_{N, L}$ and condition (ii) is satisfied.

Now let $N a \in V=V_{N . L}$, where $a=x_{1} \ldots x_{n}$ is a reduced word. Then

$$
\left(N x_{1} \ldots x_{i}, x_{i+1}, N x_{1} \ldots x_{i+1}\right) \in E_{N, L} \text {, }
$$

for all $i, 0 \leq i \leq n-1$. It follows immediately that condition (iii) is satisfied and that $\Gamma_{N, L}$ is an inverse word graph. It is obvious that condition (iv) is satisfied. The finiteness of $\Gamma_{N . L}$ follows from the fact that $X$ is finite and $L$ is the union of finitely many cosets of $N$. Thus $\Gamma_{N . L}$ is a deterministic inverse word graph.

Now let $\Gamma=(V, E)$ be an arbitrary finite deterministic inverse word graph and let $P \in V$. Let

$$
\begin{aligned}
& N=\pi(\Gamma, P), \\
& L=\{\text { reduced or empty words labelling walks from } P\} .
\end{aligned}
$$

Let $g, h \in L$. Then $g$ and $h$ label walks from $P$ ending in the same vertex

$$
\begin{aligned}
& \Leftrightarrow g h^{-1} \text { labels a walk from } P \text { to } P \\
& \Leftrightarrow g h^{-1} \in N \\
& \Leftrightarrow N g=N h .
\end{aligned}
$$

Therefore $L$ is the union of finitely many cosets of $N$. For each $Q \in V$, let $g_{Q}$ be a reduced word labelling a walk from $P$ to $Q$. Then $g_{Q} \in L$ and, by (6),

$$
\varphi: Q \rightarrow N g_{Q} \quad(Q \in V)
$$

is an injective mapping of $V$ into $V_{N, L}$. By the definition of $L$, it is clear that $\varphi$ is 
surjective. It is straightforward to verify that $\varphi$ extends a bijection of $E$ onto $E_{N . L}$ that preserves labels and therefore that $\varphi$ is an isomorphism.

Not surprisingly, there is a strong direct connection between the fundamental group and automorphism group of $\Gamma_{N . L}$ and the values of $N$ and $L$.

Theorem 5.3. Let $(N, L)$ be an inverse word pair and $\Gamma=\Gamma_{N . L}$. Let $\Phi$ be the automorphism group of $\Gamma$ and $\tau_{V}$ be the orbit relation on $V=V_{N, L}$ under $\Phi$.

(i) $\pi(\Gamma, N)=N$.

(ii) $\pi\left(\Gamma / \Phi, N \tau_{v}\right)=\bigcup\{\varphi(N): \varphi \in \Phi\}=\{g: g$ labels a walk from $N$ to $\varphi(N)$, for some $\varphi \in \Phi\}$.

(iii) $L$ is a union of finitely many cosets of $\pi\left(\Gamma / \Phi, N \tau_{V}\right)$.

(iv) For each $g \in \pi\left(\Gamma / \Phi, N \tau_{v}\right)$, define

$$
\varphi_{g}: N a \rightarrow N g a \quad\left(N a \in V_{N, L}\right)
$$

Then $\varphi_{g}$ is an automorphism of $\Gamma$ and $\Phi=\left\{\varphi_{g}: g \in \pi\left(\Gamma / \Phi, N \tau_{V}\right)\right\}$.

Proof. (i) Clearly $g \in F \mathscr{G}(X)$ labels a walk from $N$ to $N$ in $\Gamma$ if and only if $N g=N$. Thus (i) holds.

(ii) Let $H$ and $K$ denote the sets on the left and right of the first equality in (ii), respectively. Let $g \in K$, say $g \in \varphi(N)$ where $\varphi \in \Phi$. Then, since $\varphi(N)$ is a coset of $N$, we must have $\varphi(N)=N g$. Taking $g$ in reduced form, it follows that $g$ labels a walk from $N$ to $N g$ in $\Gamma$, since $L$ is a Schreier subset. But $N g=\varphi(N) \in \Phi(N)$ whence $g \in H$. Thus $K \subseteq H$.

Conversely, let $g \in H$. Then $g$, in reduced form, labels a walk from $N$ to a vertex $Q$, say in $\Phi(N)$. But if $g$ labels a walk in $\Gamma$ from $N$ then it must be from $N$ to $N g$. Thus $N g=Q \in \Phi(N)$ so that $g \in N g \subseteq K$. Hence $H \subseteq K$ and the first equality in (ii) holds.

The second equality follows immediately from the observation that if $g$ is a reduced word and $\varphi \in \Phi$, then

$$
\begin{aligned}
g \in \varphi(N) & \Leftrightarrow \varphi(N)=N g \\
& \Leftrightarrow g \text { labels a walk from } N \text { to } \varphi(N) .
\end{aligned}
$$

(iii) Let $g \in L$ and $h \in H=\pi\left(\Gamma / \Phi, N \tau_{v}\right)$. Then taking $h$ to be reduced, it follows that $h$ labels a walk from $P$ to $\varphi(P)$, for some $\varphi \in \Phi$. Since $\varphi$ is an automorphism of $\Gamma_{N, L}$ and $g$ labels a walk from $P$, it follows that $g$ labels a walk from $\varphi(P)$. Thus $h g$ labels a walk from $P$ and so does the reduced form of $h g$. Thus $h g \in L$ and $L$ is a union of cosets of $H$. Since $N \subseteq H \subseteq L$ and $(N, L)$ is an inverse word pair, $L$ must be the union of just finitely many cosets of $H$.

(iv) Let $g \in H=\pi\left(\Gamma / \Phi, N \tau_{V}\right)$ and be reduced. By the proof of Theorem 4.6, there exists a unique automorphism $\varphi_{g}$ of $\Gamma$ such that $g$ labels a walk from $N$ to $\varphi_{g}(N)$. But in $\Gamma_{N, L}$ this means that we must have

$$
\varphi_{g}(N)=N g
$$

For any $N a \in V_{N, L}$, with $a$ in reduced form, $a$ labels a walk from $N$ to $N a$ and therefore from $\varphi_{g}(N)$ to $\varphi_{g}(N a)$, that is, from $N g$ to $\varphi_{g}(N a)$. Consequently

$$
\varphi_{g}(N a)=N g a \text {. }
$$

Thus $\varphi_{g}$ is of the form described in (iv) and since, by Theorem 4.6, every automorphism is of the form $\varphi_{g}$, for some $g \in H$, the claim follows. 
TheOREM 5.4. Let $N$ be a subgroup of $F \mathscr{G}(X)$ and $L$ be a Schreier subset that is a union of finitely many cosets of $N$. Let $\Phi$ be the automorphism group of $\Gamma_{N . L}, \tau_{V}$ the orbit relation on $V$ under $\Phi$ and $H=\pi\left(\Gamma_{N, L} / \Phi, N \tau_{V}\right)$. Then the following statements are equivalent.

(i) $\Gamma_{N, L}$ is a Schützenberger graph.

(ii) $g \in F \mathscr{G}(X)$ and $g L \subseteq L \Rightarrow g \in H$.

(iii) $H$ is the maximum subsemigroup of $F \mathscr{G}(X)$ such that $H L \subseteq L$.

Proof. (i) implies (ii). Let $g \in F \mathscr{G}(X)$ and $g L \subseteq L$ and $V=V_{N . L}$. Suppose that $N g \notin \Phi(N)$. By Theorem 2.9 there exists a reduced word $w$ such that $w$ labels a walk from $N$ but not from $N g$. In other words, $N w \in V$ but $N g w \notin V$ so that $w \in L$ but $g w \notin L$, a contradiction. Therefore $N g \in \Phi(N)$ so that, by Theorem 5.3, $g \in H$.

(ii) implies (i). Let $N g \notin \Phi(N)$ where $g$ is reduced. Then, by Theorem 5.3, $g \notin H$. Therefore, by hypothesis, there exists $w \in L$ with the reduced form of $g w \notin L$. But then $w$ labels a walk from $N$ but not from $N g$. By Theorem 2.9, $\Gamma_{N, L}$ is a Schützenberger graph.

(ii) implies (iii). Let $M$ be a subsemigroup of $F \mathscr{G}(X)$ such that $M L \subseteq L$. Then, for any $g \in M$, we have

$$
g L \subseteq M L \subseteq L
$$

so that, by (ii), $g \in H$. Thus $M \subseteq H$ and (iii) holds.

(iii) implies (ii). Let $g \in F^{\mathscr{G}}(X)$ be such that $g L \subseteq L$. Then $g^{k} L \subseteq L$, for all $k \geq 0$, and so $\langle g\rangle L \subseteq L$. By (iii), this implies that $\langle g\rangle \subseteq H$ so that $g \in H$ and (ii) holds.

6. Coverings. Let $\Gamma=(V, E)$ be an inverse word graph and $P \in V$. We define

$$
\operatorname{Star}(P)=\{(P, x, v):(P, x, v) \in E\} .
$$

Let $\Gamma=(V, E)$ and $\Delta=(W, F)$ be deterministic inverse word graphs. By a covering morphism $\theta$ of $\Gamma$ to $\Delta$ is meant a morphism which, for each $u \in V$, induces a bijection of $\operatorname{Star}(P)$ onto $\operatorname{Star}(\theta P)$ for all $P \in V$. If there is a covering morphism from $\Gamma$ to $\Delta$, then we say that $\Gamma$ covers $\Delta$. For a general treatment of covering spaces, we refer the reader to [2], Chapter 6.

Note that, if $P \in V$ and $Q \in W$, then there can be at most one covering morphism $\theta$ such that $\theta P=Q$. In addition, any covering morphism must be surjective, since any deterministic inverse word graph is connected.

THeORem 6.1. Let $(M, K)$ and $(N, L)$ be inverse word pairs. Then there exists a covering morphism $\theta: \Gamma_{M . K} \rightarrow \Gamma_{N . L}$, with $\theta M=N$, if and only if

(i) $K=L$, and

(ii) $M \subseteq N$.

If $\theta$ is such a covering then

$$
\theta(\operatorname{Ma}, x, \operatorname{Max})=(N a, x, N a x) \quad\left(a, a x \in L, x \in X \cup X^{-1}\right) .
$$

Proof. Let $\theta: \Gamma_{M, K} \rightarrow \Gamma_{N . L}$ be a graph morphism with $\theta M=N$. Let $m$ be a reduced word in $M$. Then $m$ labels a walk from $M$ to $M$. Hence $m$ must also label a walk from $N$ to $N$, in other words, $N m=N$. Thus $m \in N$ and $M \subseteq N$. In the same way, any word $a$ that labels a walk in $\Gamma_{M, K}$ from $M$ must label a walk in $\Gamma_{N, L}$ from $N$, since $\theta$ is a graph 
morphism with $\theta M=N$. Thus $K \subseteq L$. Conversely, any word that labels a walk in $\Gamma_{N, L}$ from $N$ must label a walk in $\Gamma_{M, K}$ from $M$ since $\theta$ is a covering morphism. Hence $L \subseteq K$ and equality holds.

Now let (i) and (ii) hold and define $\theta$ as in (7). By (ii), $\theta$ is well defined and, by (i), $\theta$ is a graph morphism. Consider any vertex $M a, a \in K$. Then the edges emanating from $M a$ are precisely those of the form

$$
(\operatorname{Ma}, x, \operatorname{Max}) \quad(a, a x \in K) .
$$

Now $\theta(M a)=N a$ and $a x \in K=L$ so that

$$
\theta(\operatorname{Ma}, x, \operatorname{Max})=(N a, x, N a x) .
$$

Since the label remains the same and both graphs are deterministic, it follows that $\theta$ is injective on $\operatorname{Star}(M a)$. On the other hand, if

then $a, a x \in L=K$ so that

$$
(N a, x, N a x) \in E\left(\Gamma_{N, L}\right)
$$

$$
(M a, x, M a x) \in E\left(\Gamma_{M, L}\right)
$$

and

$$
\theta(\operatorname{Ma}, x, M a x)=(N a, x, N a x) .
$$

Thus $\theta$ induces a bijection of $\operatorname{Star}(\mathrm{Ma})$ onto $\operatorname{Star}(\mathrm{Na})$ and $\theta$ is a covering morphism.

From the remark prior to the theorem, there can be only one covering morphism with $\theta M=N$ which must, by the above, be the one given in (7).

For any Schreier subset $L$ of $F \mathscr{G}(X)$, it is evident that $(H, L)$ will be an inverse word pair whenever $H$ is a subgroup and $L$ is the union of finitely many cosets of $H$. What is not so clear is when there will exist a subgroup $N$ such that $\Gamma_{N, L}$ is a Schützenberger graph.

THEOREM 6.2. Let $L$ be a Schreier subset of $F \mathscr{G}(X)$. Let $F=\{g \in F \mathscr{G}(X): g L \subseteq L\}$. Then the following statements are equivalent. graph.

(i) There exists a subgroup $N$ of $F \mathscr{G}(X)$ such that $\Gamma_{N, L}$ is a finite Schützenberger

(ii) $F$ is a subgroup and $L$ is a union of a finite number of cosets of $F$.

Proof. (i) implies (ii). Let $N$ be as in (i) and let $\Phi$ denote the automorphism group of $\Gamma_{N, L}$. By Theorem 5.4(iii), $F=\pi\left(\Gamma_{N, L} / \Phi, N \tau_{V}\right)$, whence $F$ is a subgroup. By Theorem 5.3(iii), $L$ is the union of finitely many cosets of $F$. Thus (ii) holds.

(ii) implies (i). Directly from the hypothesis we know that $(F, L)$ is an inverse word pair. Let $\Phi$ denote the automorphism group of $\Gamma=\Gamma_{F, L}$ and $\tau_{V}$ be the orbit relation on $V=V_{F, L}$ under $\Phi$. By Theorem 5.3(iii), $L$ is a union of cosets of $H=\pi\left(\Gamma / \Phi, F \tau_{V}\right)$ so that $H L \subseteq L$ and therefore $H \subseteq F$. Then

$$
\begin{array}{rlrl}
F & =\pi(\Gamma, F), & & \text { by Theorem } 5.3(\mathrm{i}), \\
& \subseteq H, & \text { by Theorem 4.6(i). }
\end{array}
$$

Hence $H=F$ and, by Theorem 5.4(iii), $\Gamma$ is a Schützenberger graph. 
DefinItION 6.3. In the light of Theorem 6.2, we will say that $L$ is a Schützenberger subset of $F \mathscr{G}(X)$ if the following conditions are satisfied

(i) $L$ is a Schreier subset of $F \mathscr{G}(X)$;

(ii) $F=\left\{g \in F^{\mathscr{G}}(X): g L \subseteq L\right\}$ is a subgroup of $L$;

(iii) $L$ is the union of finitely many cosets of $F$.

In other words, $L$ is a Schützenberger subset of $F \mathscr{G}(X)$ if and only if there exists a subgroup $N$ of $F \mathscr{G}(X)$ such that $\Gamma_{N . L}$ is a finite Schützenberger graph.

The next observation follows immediately from Theorem 6.1.

Lemma 6.4. Let $L$ be a Schützenberger subset of $F \mathscr{G}(X)$ and $F=\{g \in F \mathscr{G}(X): g L \subseteq$ $L\}$. Then $\Gamma_{N . L}$ covers $\Gamma_{F, L}$, for every subgroup $N$ of finite index in $F$.

This leads naturally to the question of which subgroups of $F$ correspond to covers of $\Gamma_{F, L}$ that are also Schützenberger graphs.

Proposition 6.5. Let $L$ be a Schützenberger subset of $F \mathscr{G}(X)$ and $F=\{g \in$ $F \mathscr{G}(X): g L \subseteq L\}$. Let $N$ be a subgroup of $F$ of finite index. Then $\Gamma_{N, L}$ is a Schützenberger graph if and only if $N$ is normal in $F$.

Proof. Suppose that $\Gamma=\Gamma_{N, L}$ is a Schützenberger graph with automorphism group $\Phi$ and orbit relation $\tau_{V}$ on $V=V_{N . L}$. By Theorem $4.6, N=\pi(\Gamma, N)$ is a normal subgroup of $\pi\left(\Gamma / \Phi, N \tau_{V}\right)$ which, by Theorem $5.4($ iii) is the same as $F$. This establishes the direct implication.

Now suppose that $N$ is normal in $F$. Let $\Phi$ denote the automorphism group of $\Gamma=\Gamma_{N, L}$ and let $\tau_{V}$ denote the orbit relation of $\Phi$ on $V$. Let

$$
H=\pi\left(\Gamma_{N . L} / \Phi, N \tau_{V}\right) \text {. }
$$

By Theorem 5.4(iii), we must show that $H=F$. By Theorem 5.3(ii), this is equivalent to showing that

$$
F=\bigcup\{\varphi(N): \varphi \in \Phi\} .
$$

Let $K$ denote the right hand side of (8). Let $g \in L \backslash F$. Then $g L$ is not contained in $L$ so that $g a \notin L$, for some $a \in L$. Therefore $a$ labels a walk from $N$ but not from $N g$. Consequently $N g \neq \varphi(N)$, for any $\varphi \in \Phi$. Hence $K \subseteq F$.

Now let $g \in F$. Then $g L \subseteq L$ and any element $a \in L$ labels a walk from $N g$. Hence the mapping

$$
\varphi:(N a, x, N a x) \rightarrow(N g a, x, N g a x)
$$

determines a morphism which is well defined, since $N$ is a normal subgroup of $F$. Furthermore, if

$$
\varphi(N a, x, N a x)=\varphi(N b, y, N b y)
$$

then $N g a=N g b$ and $x=y$. But

$$
\begin{aligned}
N g a=N g b & \Leftrightarrow g N a=g N b \quad \text { (since } N \text { is normal in } F) \\
& \Leftrightarrow N a=N b .
\end{aligned}
$$

Thus $\varphi$ is one-to-one and, since $\Gamma_{N, L}$ is finite, $\varphi$ is an automorphism. Since $\varphi$ maps $N$ to $N g$, it follows that $N g \subseteq K$. Thus $g \in K$ and so $F \subseteq K$. Therefore $F=K$, as required. 


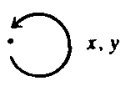

Figure 3.

Lemma 6.4 and Proposition 6.5 reveal that there can be inverse word graphs that cover a Schützenberger graph that are not themselves Schützenberger graphs. A very simple example that illustrates this fact is the graph in Figure 2 . It is easily verified that $\Gamma$ covers the graph in Figure 3, which is a Schützenberger graph.

However, as observed previously, $\Gamma$ is not a Schützenberger graph. This example is, in fact, doubly interesting since there is no way of labelling $\Gamma$ in order to make it a Schützenberger graph over $\{x, y\}$.

\section{REFERENCES}

1. A. H. Clifford and G. B. Preston, The algebraic theory of semigroups, Vol. 1, Amer. Math. Soc. Surveys, No. 7. 1961.

2. D. E. Cohen, Combinatorial Group Theory: a topological approach, London Math. Soc. Student Texts, Cambridge University Press, Cambridge, U.K.

3. S. W. Margolis and J. Meakin, E-unitary inverse monoids and the Cayley graph of a group presentation, J. Pure Appl. Algebra 58 (1989), 45-76.

4. S. W. Margolis and J. Meakin, Graph immersions and inverse monoids, in Monoids and semigroups with applications, ed. J. Rhodes (World Scientific, 1991), 144-158.

5. S. W. Margolis and J. Meakin, Immersions and the free inverse category over a graph (manuscript).

6. S. W. Margolis and J. Meakin, Free inverse monoids and graph immersions, International J. Algebra and Computation 3 (1993), 79-100.

7. S. W. Margolis, J. Meakin and P. Jones, Free products of inverse semigroups II Glasgow Math. J. 33 (1991), 373-387.

8. J.-P. Serre, Trees (Springer-Verlag, 1980).

9. J. B. Stephen, Presentation of inverse monoids, J. Pure Appl. Algebra 63 (1990), 81-112.

Department of Mathematics and Statistics

SimON Fraser UNIVERSITY

British Columbia

CANADA 